\section{Uso regular de serviços odontológicos entre adultos: padrões de utilização e tipos de serviços}

\author{
Regular use of dental care services by adults: \\ patterns of utilization and types of services
}

\author{
1 Programa de Pós-graduação \\ em Epidemiologia, \\ Universidade Federal de \\ Pelotas, Pelotas, Brasil. \\ Correspondência \\ M. B. J. Camargo \\ Programa de Pós-graduação \\ em Epidemiologia, \\ Universidade Federal de \\ Pelotas. \\ Rua Marechal Deodoro 1160 \\ 3 o piso, Pelotas, $R S$ \\ 96020-220, Brasil. \\ mbiajac@uol.com.br
}

\section{Abstract}

The aim of this study was to estimate the prevalence of regular use of dental services by adults and identify groups where this behavior is more frequent. A cross-sectional population-based study was carried out in Pelotas, southern Brazil, including 2,961 individuals who answered a standardized questionnaire. Overall prevalence of regular use of dental services was $32.8 \%$. The following variables were positively associated with regular use: female gender, age $\geq 60$ years, no partner, high educational level, high economic status, private service user, good/excellent self-rated oral health, and no perceived need for dental treatment. Those who had received orientation on prevention and expressed a favorable view towards the dentist had higher odds of being regular users. Especially among lower-income individuals, regular use was infrequent (15\%). When restricting the analysis to users of public dental services, schooling was still positively associated with the outcome. Dental services, especially in the public sector, should develop strategies to increase regular and preventive use.

Dental Care; Health Services; Adult
Maria Beatriz J. Camargo ${ }^{1}$

Samuel C. Dumith 1

Aluísio J. D. Barros 1

\section{Introdução}

Nos últimos vinte anos, a experiência de cárie entre escolares no Brasil teve um relevante declínio, podendo-se observar uma redução de $61,7 \%$ aos 12 anos de idade. Esta redução se deu principalmente pela elevação no acesso à água e creme dental fluorados e programas de saúde bucal coletiva direcionados a essa faixa etária. Entretanto, a condição de saúde bucal permaneceu praticamente inalterada entre os adultos e idosos 1 . Pesquisa realizada em 1998 demonstrou que o serviço público respondeu por apenas $24 \%$ dos atendimentos odontológicos, sendo necessário ampliar o acesso aos serviços de saúde 2 .

Com o intuito de requalificar a atenção em saúde bucal e ampliar o acesso aos serviços, o Ministério da Saúde, a partir de 2000, estabeleceu incentivo financeiro para a inserção das ações de saúde bucal. Para tal, efetuou a contratação de cirurgião-dentista, atendente de consultório dentário e técnico de higiene dentária nas equipes do Programa Saúde da Família (PSF), criando as equipes de saúde bucal. A inclusão desses profissionais vem ao encontro do princípio da integralidade, segundo o qual todas as necessidades em saúde dos indivíduos deveriam ser supridas.

Esse processo de requalificação da atenção básica está em permanente construção e se apresenta em estágios diferentes entre regiões e municípios brasileiros 3 . Dificuldades em se trabalhar em equipe, capacitação dos profissionais 
para atuar na perspectiva de um novo modelo de atenção, no qual a educação em saúde tenha um importante papel na melhora do autocuidado dos indivíduos, enfatizando procedimentos preventivos, têm sido relatadas por diversos pesquisadores como desafios a serem enfrentados para a consolidação da odontologia no Sistema Único de Saúde (SUS) 4,5.

Nesse contexto, a utilização dos serviços odontológicos de forma regular pode proporcionar um maior contato do paciente com o dentista, fazendo com que questões como importância do autocuidado, hábitos nocivos à saúde e conhecimento sobre as doenças bucais possam ser trabalhadas 6 . Além disso, cáries dentárias e problemas periodontais poderiam ser detectados precocemente, exigindo procedimentos de menor complexidade.

Embora não se tenha consenso sobre um intervalo ideal entre as consultas odontológicas ?, muitos estudos têm demonstrado benefícios da utilização regular de serviços odontológicos $6,8,9,10,11$. Pesquisadores observaram que usuários regulares apresentavam melhor percepção de saúde bucal 8 , menos perdas dentárias 10 , melhor condição gengival (melhor controle de placa e menor acúmulo de cálculo) 8,9,10,11 e menor probabilidade de sentir dor de dente 9 . Todos esses fatores, idealmente, poderiam colaborar para manutenção da saúde bucal (estética e função) por mais tempo ao longo da vida.

Em uma análise entre a quantidade de procedimentos preventivos realizados pelo serviço público e a presença de dentes cariados, em uma amostra de jovens do Rio Grande do Sul, com idade entre 15 e 19 anos, pôde-se concluir que, após controle para variáveis sócio-econômicas, aqueles que moravam em municípios com as menores taxas de procedimentos preventivos tiveram maior chance de apresentar dentes cariados 12. Tais procedimentos são mais comumente realizados em indivíduos que buscam o serviço de forma regular, e não em indivíduos que o procuram apenas quando estão com dor ou quando a doença já progrediu a ponto de apresentar desconforto 13 .

De nosso conhecimento, no Brasil, foi realizado apenas um estudo de base populacional sobre as características associadas ao uso regular de serviços odontológicos 14. Esse estudo foi realizado em um pequeno município no Estado de Minas Gerais, encontrando prevalência de uso regular de $24,6 \%$, e as características associadas positivamente ao desfecho foram: ter escolaridade igual ou superior a 4 anos, preferir tratamento dentário restaurador a extração e acreditar que ir ao dentista previne a cárie e a doença na gengiva.
Encontrou-se um outro estudo realizado com funcionários de uma universidade pública brasileira, porém o uso regular foi tratado como uma variável explanatória ${ }^{8}$.

O objetivo do presente estudo foi avaliar a prevalência de uso regular de serviços odontológicos e as características dos grupos com maior utilização, identificando variáveis facilitadoras desse tipo de utilização.

\section{Métodos}

Este estudo foi realizado no Município de Pelotas, Rio Grande do Sul, Brasil. A cidade localiza-se no extremo sul do país e conta com aproximadamente 340 mil habitantes, dos quais cerca de 95\% reside na zona urbana (Instituto Brasileiro de Geografia e Estatística. Censo demográfico, 2000: estimativa para 2007. http://www.ibge.gov. $\mathrm{br} /$ cidadesat/topwindow.htm? 1, acessado em 10/Abr/2009). Do ponto de vista econômico, as principais atividades do município são agricultura, pecuária e comércio.

Foi realizado um inquérito populacional envolvendo 14 mestrandos, no qual vários aspectos relacionados à saúde da população foram concomitantemente investigados em uma amostra de adultos, incluindo a utilização dos serviços odontológicos de forma regular 15 . O processo de amostragem foi realizado em dois estágios, utilizando os setores censitários definidos pelo Censo Demográfico de 2000 do Instituto Brasileiro de Geografia e Estatística (IBGE, http://www. ibge.gov.br) como unidade amostral primária, $\mathrm{e}$ os domicílios, como unidade secundária.

A partir de dados da literatura 14, empregouse uma prevalência estimada de $25 \%$ para utilização dos serviços odontológicos de forma regular. Tolerando-se um erro de três pontos percentuais, a um nível de $95 \%$ de confiança, obteve-se um tamanho de amostra de 800 indivíduos. Ajustando-se esse número para o efeito do delineamento amostral esperado (EDA) de 2,5, e mais um acréscimo de $10 \%$ para possíveis perdas e recusas, o tamanho da amostra necessário passou a ser de 2.200 indivíduos. O estudo teve um poder de $80 \%$ para detectar razão de prevalência (RP) de pelo menos 1,4, com proporção de exposição variando de $30 \%$ a $84 \%$, considerando o acréscimo do EDA $(2,5)$, perdas $(10 \%)$ e fatores de confusão (15\%).

Houve a necessidade de se obter um tamanho de amostra que contemplasse todos os desfechos estudados pelos 14 mestrandos, totalizando-se, assim, 1.440 domicílios distribuídos em 126 setores censitários, dos 404 setores residenciais existentes na cidade. Os setores cen- 
sitários foram selecionados sistematicamente, com probabilidade proporcional ao tamanho e estratificados em relação à renda média do chefe da família, com base nos dados do Censo Demográfico de 2000. Em cada setor selecionado foi feita a enumeração dos domicílios particulares permanentes, e o número original de domicílios desejados foi ajustado para a variação observada em relação ao número de domicílios presentes por ocasião do censo. Dentro de cada setor, foram selecionados, por amostragem sistemática, 1.534 domicílios (média de 12 por setor). Todos os moradores com idade igual ou superior a 20 anos capazes de responder o questionário foram considerados elegíveis para o estudo.

Os dados foram coletados nos domicílios por entrevistadoras treinadas pelos mestrandos, as quais compareciam três vezes aos endereços selecionados antes de aceitarem uma perda ou recusa. A coleta de dados foi realizada de 23 de outubro de 2007 a 15 de janeiro de 2008.

Para obtenção do desfecho - usar o serviço de forma regular -, quatro opções de rotinas de consultas foram dadas aos entrevistados 16: (1) "Eu nunca vou ao dentista"; (2) "Eu vou ao dentista quando eu tenho dor ou quando eu tenho um problema nos meus dentes ou na gengiva"; (3) "Eu vou ao dentista às vezes, tendo um problema ou não"; (4) "Eu vou ao dentista de forma regular". Foram considerados usuários regulares aqueles que escolheram a opção três ou quatro.

As variáveis utilizadas para este estudo foram baseadas na classificação proposta por Andersen 17 e Andersen \& Newman ${ }^{18}$. Segundo os autores, a utilização dos serviços de saúde que incluem os serviços odontológicos depende das características da população em risco e dos serviços de saúde utilizados. Essas características estão divididas em três grupos: variáveis de predisposição, facilitação e necessidade. No primeiro grupo, a predisposição do indivíduo para usar os serviços de saúde inclui variáveis sócio-demográficas (idade, sexo, estado conjugal, cor da pele e escolaridade), atitudes e opiniões (valor dado à saúde, conhecimento sobre a doença e opinião sobre o tratamento). Ter plano de saúde e acesso a fonte regular de cuidado odontológico compõe o grupo facilitação ao uso dos serviços. No grupo de necessidades, estão incluídas a autopercepção de necessidade de tratamento e o diagnóstico por profissional.

As variáveis de predisposição utilizadas neste estudo foram: sexo (masculino e feminino), faixa etária (de 20-39, 40-59 e $\geq 60$ anos de idade), escolaridade $(\leq 4,5-8,9-11$ e $\geq 12$ anos completos de estudo) e situação conjugal (com companheiro e sem companheiro). Como variáveis de conhecimentos e opiniões, foram utilizadas as seguintes questões: 1) "Dentistas recomendam mais tratamento do que eu necessito", para captar uma opinião favorável em relação ao profissional e ao tratamento; 2) “Todo mundo perde os dentes com o passar da idade" e "Salvar um dente da frente é mais importante que salvar um dente de trás", para obter informação a respeito de crenças sobre o fatalismo de se tornar edêntulo e dar uma maior importância ao componente estético da saúde bucal. A autopercepção de saúde bucal também foi investigada (categorizada em: ótima, boa, regular, ruim e péssima). Para captar o conhecimento sobre prevenção de doenças bucais, foi perguntado se os indivíduos, alguma vez na vida, haviam recebido orientações do cirurgiãodentista sobre como evitar problemas nos dentes e gengivas ( $\operatorname{sim}$ ou não).

As variáveis de facilitação foram nível econômico (utilizando os pontos da Associação Brasileira de Empresas de Pesquisa -ABEP 19 e categorizados em quintis) e tipo de serviço procurado habitualmente (público, convênio ou particular). Para a análise da utilização do serviço odontológico, as categorias convênio e particular foram agrupadas em serviço privado por não apresentarem diferenças estatísticas significativas.

Como variável de necessidade, foi utilizada a autopercepção de necessidade de tratamento. Para ampliar a capacidade de entendimento desta variável, ela foi categorizada em uma escala de quatro pontos 20: "Sim, para fazer uma revisão"; "Não, está tudo bem com os meus dentes"; "Sim, estou com dor ou tenho um problema para resolver"; "Não, eu tenho um problema, mas ele pode esperar".

A análise ajustada foi realizada de acordo com um modelo hierárquico de determinantes. As variáveis foram distribuídas em três níveis: no distal estão as variáveis demográficas e sócio-econômicas (sexo, idade, escolaridade, classe social, cor da pele e situação conjugal); no intermediário as variáveis relacionadas ao uso de serviços e autopercepção de saúde (tipo de serviço procurado e autopercepção de saúde bucal) e, no mais proximal, as variáveis de conhecimentos (ter recebido orientação sobre prevenção, acreditar que dentistas recomendam mais tratamento do que necessita, todo mundo perde os dentes com o passar da idade e salvar um dente da frente é mais importante que salvar um dente de trás). A análise foi realizada de forma que o efeito das variáveis está controlado para aquelas do mesmo nível e as dos níveis superiores.

O instrumento utilizado foi um questionário pré-codificado e previamente testado, para checar a compreensão das perguntas, em um setor que não foi incluído na amostra. Visando a garantir a qualidade dos dados, $10 \%$ dos indivíduos 
foram visitados novamente e responderam a um questionário simplificado, monitorando assim o trabalho das entrevistadoras.

O banco de dados foi obtido mediante dupla digitação e posterior checagem de inconsistências, utilizando-se o programa Epi Info versão 6.04 (Centers for Disease Control and Prevention, Atlanta, Estados Unidos). As diferenças entre o uso de forma regular e as variáveis estudadas foram testadas empregando-se o teste quiquadrado. Na análise bivariável, calcularam-se as RP brutas e seus respectivos intervalos de 95\% de confiança (IC95\%), para testar a força da associação entre o desfecho e as variáveis explicativas. Para examinar o efeito independente de cada variável, foi utilizada a regressão de Poisson, uma vez que o desfecho era binário e freqüente 21 , valendo-se do modelo conceitual descrito acima. Para cada nível, realizou-se seleção de variáveis para trás, descartando-se aquelas com $p>0,2$. As variáveis que obtiveram um $\mathrm{p} \geq 0,05 \mathrm{e}<0,2$ foram mantidas no modelo para controle de um possível efeito residual de confusão. O pacote estatístico Stata versão 9 (Stata Corp., College Station, Estados Unidos) foi empregado para as análises, que levaram em consideração o desenho amostral (com a utilização do prefixo $s v y$ ).

O estudo foi aprovado pelo Comitê de Ética e Pesquisa da Universidade Federal de Pelotas. Após esclarecimento dos objetivos gerais do trabalho e dos procedimentos envolvidos, aqueles que concordaram em participar assinaram o termo de consentimento livre e esclarecido.

\section{Resultados}

Dos 3.155 indivíduos elegíveis, foram entrevistados 2.961, atingindo uma taxa de resposta de $94 \%$. Não participaram do estudo $8 \%$ dos homens e 4,7\% das mulheres selecionadas.

A descrição da amostra e as análises brutas podem ser observadas na Tabela 1 . A população estudada, na sua maioria, era branca, predominantemente do sexo feminino e morava com companheiro(a). Em relação ao tipo de serviço procurado, aproximadamente um quarto utilizou algum tipo de convênio (foram incluídas nesta categoria: sindicatos, cirurgiões-dentistas que trabalhavam em empresas e convênios formais). Os serviços público e particular tiveram prevalências muito semelhantes, $36,7 \%$ e $37,1 \%$, respectivamente. Em relação à escolaridade, $75 \%$ dos indivíduos tinham mais de cinco anos de escolaridade. Aproximadamente 30\% não receberam orientações sobre prevenção, e $40 \%$ relataram não precisar de tratamento odontológico.
A prevalência de uso regular de serviço odontológico foi de 32,8\% (IC95\%: 30,0-35,7), e para este desfecho foi obtido um coeficiente de correlação intraclasse de 0,07, gerando um EDA de 2,8 para o desenho do estudo.

Todas as características estudadas apresentaram associação estatisticamente significativa com o uso de serviço regular na análise bruta (Tabela 1).

Mulheres, categorias de idade igual ou superior a 60 anos, indivíduos sem companheiro e aqueles que utilizaram algum tipo de convênio apresentaram maior uso do serviço. Aqueles que classificaram sua saúde bucal como ótima tiveram prevalência quatro vezes maior quando comparados aos que classificaram como ruim/ péssima. Indivíduos com escolaridade e nível econômico mais alto apresentaram maiores prevalências de uso regular, o mesmo se dando com aqueles que tinham opinião favorável em relação ao profissional e não eram fatalistas em relação ao edentulismo. Menor utilização de forma regular pôde ser observada por indivíduos que deram maior ênfase ao componente estético da saúde bucal (Tabela 1).

Na Figura 1, é apresentada a prevalência de uso regular de serviço odontológico estratificada por sexo e idade, podendo-se observar que homens e mulheres jovens tiveram prevalência de uso de forma regular muito semelhante, respectivamente $39,1 \%$ e $42,5 \%(p=0,2)$. Entretanto, as mulheres entre 40-59 e $\geq 60$ anos de idade utilizaram aproximadamente $40 \%$ mais os serviços de forma regular quando comparadas aos homens das mesmas faixas etárias.

A Tabela 2 mostra a utilização de serviço odontológico público e privado (convênio e particular), conforme algumas características estudadas. Pode-se observar que as mulheres estiveram em maior proporção de utilização no serviço público. No serviço privado, o uso de forma regular foi o dobro quando comparado ao serviço público, e os indivíduos tiveram melhor percepção de saúde bucal. Com relação à percepção de necessidade de tratamento, verificou-se, dentre aqueles que procuraram o serviço privado, uma diferença de aproximadamente dez pontos percentuais de indivíduos que disseram não necessitar de tratamento quando comparados aos que procuraram o serviço público.

O nível econômico dos indivíduos que procuraram o serviço público foi menor quando comparado ao do grupo que procurou o serviço privado. Neste, a proporção de indivíduos que manifestaram opinião favorável em relação ao profissional, que não acreditavam na fatalidade de ficar edêntulos e não davam maior ênfase ao componente estético da saúde bucal foi maior 
Tabela 1

Descrição da amostra, prevalências e razões de prevalências (RP) brutas do uso regular de serviço odontológico entre as categorias das variáveis independentes. Pelotas, Rio Grande do Sul, Brasil, 2008.

\begin{tabular}{|c|c|c|c|c|}
\hline Variáveis [N] & n (\%) & $\begin{array}{c}\text { Prevalências de uso regular } \\
\text { \% (IC95\%) }\end{array}$ & RP & Valor de $p$ \\
\hline Sexo [2.961] & & & & $<0,001$ \\
\hline Masculino & $1.276(43,1)$ & $29,6(26,3-33,1)$ & 1,00 & \\
\hline Feminino & $1.685(56,9)$ & $35,3(32,3-38,4)$ & 1,19 & \\
\hline Idade (anos) [2.961] & & & & $<0,001 * *$ \\
\hline $20-39$ & $1.263(42,7)$ & $40,9(37,2-44,8)$ & 2,31 & \\
\hline $40-59$ & $1.111(37,5)$ & $31,6(27,7-35,7)$ & 1,78 & \\
\hline$\geq 60$ & $587(19,8)$ & $17,7(14,5-21,5)$ & 1,00 & \\
\hline Cor da pele [2.957] & & & & $<0,001$ \\
\hline Branca & $2.234(75,6)$ & $35,4(32,1-38,8)$ & 1,00 & \\
\hline Preta & $402(13,6)$ & $26,4(22,2-31,1)$ & 0,74 & \\
\hline Parda & $208(7,0)$ & $25,0(19,2-31,9)$ & 0,71 & \\
\hline Outros & $113(3,8)$ & $19,5(12,9-28,2)$ & 0,55 & \\
\hline Escolaridade (anos) [2.960] & & & & $<0,001 * \star$ \\
\hline$\leq 4$ & $691(23,3)$ & $11,3(9,1-14,0)$ & 1,00 & \\
\hline $5-8$ & $932(31,5)$ & $25,3(22,5-28,3)$ & 1,97 & \\
\hline $9-11$ & $761(25,7)$ & $41,3(37,7-44,9)$ & 3,20 & \\
\hline$\geq 12$ & $576(19,5)$ & $59,7(54,7-64,6)$ & 4,64 & \\
\hline Nível econômico (quintis) * [2.930] & & & & $<0,001$ ** \\
\hline Q1 (mais pobres) & $621(21,1)$ & $15,0(12,2-18,2)$ & 1,00 & \\
\hline Q2 & $667(22,9)$ & $26,7(23,6-30,1)$ & 1,78 & \\
\hline Q3 & $549(18,7)$ & $33,2(28,5-38,1)$ & 2,21 & \\
\hline Q4 & $547(18,7)$ & $41,1(35,3-47,3)$ & 2,74 & \\
\hline Q5 (mais ricos) & $546(18,6)$ & $53,0(46,8-58,9)$ & 3,53 & \\
\hline Situação conjugal [2.959] & & & & 0,02 \\
\hline Com companheiro & $1.806(61,0)$ & $31,1(27,9-34,4)$ & 1,00 & \\
\hline Sem companheiro & $1.153(39,0)$ & $35,7(32,1-39,3)$ & 1,15 & \\
\hline Tipo de serviço procurado [2.890] & & & & $<0,001$ \\
\hline Público & $1.061(36,7)$ & $19,7(17,3-22,3)$ & 1,00 & \\
\hline Particular & $1.073(37,1)$ & $40,3(36,3-44,3)$ & 2,04 & \\
\hline Convênio & $756(26,2)$ & $43,0(38,7-47,4)$ & 2,28 & \\
\hline Autopercepção de saúde bucal [2.961] & & & & $<0,001 * *$ \\
\hline Ótima & $421(14,2)$ & $52,3(46,6-57,8)$ & 4,24 & \\
\hline Boa & $1.305(44,1)$ & $38,8(35,4-42,3)$ & 3,14 & \\
\hline Regular & $943(31,8)$ & $22,3(19,5-25,3)$ & 1,81 & \\
\hline Ruim/Péssima & $292(9,9)$ & $12,3(8,2-18,1)$ & 1,00 & \\
\hline Necessidade de tratamento [2.959] & & & & $<0,001$ \\
\hline Sim, para revisão & $661(22,3)$ & $46,1(41,3-51,1)$ & 2,35 & \\
\hline Não, está ok & $1.181(40,0)$ & $34,6(30,9-38,4)$ & 1,76 & \\
\hline Sim, tenho problemas & $827(27,9)$ & $24,3(21,0-27,9)$ & 1,24 & \\
\hline Não, pode esperar & $290(9,8)$ & $19,7(15,3-24,9)$ & 1,00 & \\
\hline Recebeu orientação sobre prevenção [2.878] & & & & $<0,001$ \\
\hline Sim & $2.071(72,0)$ & $42,3(39,3-45,4)$ & 3,67 & \\
\hline Não & $807(28,0)$ & $11,5(9,3-14,2)$ & 1,00 & \\
\hline \multicolumn{5}{|l|}{ Dentistas recomendam mais tratamento que o } \\
\hline necessário [2.961] & & & & $<0,001$ \\
\hline Concorda & $737(24,9)$ & $23,2(20,1-26,6)$ & 1,00 & \\
\hline Não concorda & $1.766(59,6)$ & $41,7(38,3-45,3)$ & 1,80 & \\
\hline Não sabe & $458(15,5)$ & $14,0(11,1-17,4)$ & 0,60 & \\
\hline
\end{tabular}

(continua) 


\begin{tabular}{|c|c|c|c|c|}
\hline Variáveis [N] & n (\%) & $\begin{array}{c}\text { Prevalências de uso regular } \\
\qquad \%(I C 95 \%)\end{array}$ & $\mathrm{RP}$ & Valor de $\mathrm{p}$ \\
\hline \multicolumn{5}{|c|}{ Todo mundo perde os dentes com o passar } \\
\hline do tempo $[2.961]$ & & & & $<0,001$ \\
\hline Concorda & $1.307(44,1)$ & $24,6(21,8-27,6)$ & 1,00 & \\
\hline Não concorda & $1.436(48,5)$ & $41,6(38,2-45,2)$ & 1,70 & \\
\hline Não sabe & $218(7,4)$ & $24,3(19,2-30,2)$ & 0,99 & \\
\hline \multicolumn{5}{|c|}{ Salvar os dentes da frente é mais importante } \\
\hline que salvar os dentes de trás [2.961] & & & & $<0,001$ \\
\hline Concorda & $1.560(52,6)$ & $26,4(23,5-29,0)$ & 1,00 & \\
\hline Não concorda & $1.254(42,4)$ & $41,6(37,9-45,3)$ & 1,59 & \\
\hline Não sabe & $147(5,0)$ & $29,3(21,9-37,9)$ & 1,12 & \\
\hline
\end{tabular}

IC95\%: intervalo de 95\% de confiança.

* De acordo com os critérios da Associação Brasileira de Empresas de Pesquisa 20.

** Teste de tendência linear.

Figura 1

Prevalência de uso regular de serviço odontológico por faixa etária entre homens ( $N=1.276)$ e mulheres ( $N=1.685)$. Pelotas, Rio Grande do Sul, Brasil, 2008.

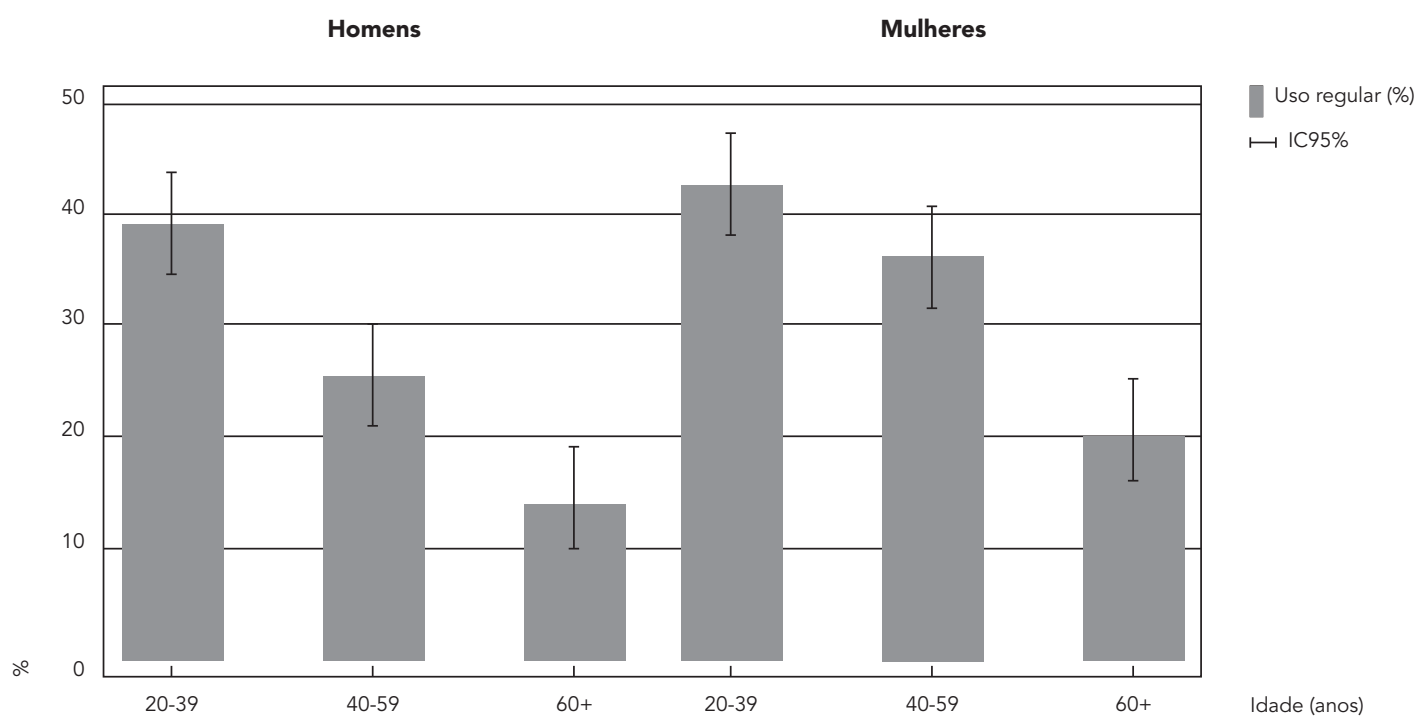

Diferença entre homens e mulheres conforme faixa etária: $20-39$ ( $p=0,2), 40-59(p<0,001)$ e 60+ $(p=0,04)$.

quando comparado ao serviço público. Ao se compararem indivíduos que não receberam orientação sobre como prevenir doenças bucais, aqueles que procuraram o serviço público apresentaram freqüência duas vezes maior do que os que utilizaram o serviço privado.

Na Figura 2, pode-se observar que, entre os menos escolarizados, a prevalência de usuários regulares foi semelhante, para os que procuram tanto o serviço público, quanto o serviço privado. No entanto, entre os mais escolarizados, esta prevalência foi aproximadamente duas vezes maior no serviço privado.

Na análise multivariável, as categorias que se mantiveram associadas de forma independente ao uso regular de serviço odontológico foram as seguintes (Tabela 3): mulheres, categorias de idade igual ou superior a 60 anos, indivíduos 
Tabela 2

Perfil dos usuários conforme o tipo de serviço odontológico utilizado. Pelotas, Rio Grande do Sul, Brasil, 2008.

\begin{tabular}{|c|c|c|c|}
\hline \multirow[t]{3}{*}{ Variáveis } & \multicolumn{2}{|c|}{ Tipo de serviço que procura } & \multirow{3}{*}{ Valor de $p$ ** } \\
\hline & Público ( $n=1.061)$ & Privado * $(n=1.829)$ & \\
\hline & $\%(I C 95 \%)$ & $\%(I C 95 \%)$ & \\
\hline Forma de uso & & & $<0,001$ \\
\hline Regular & $19,7(17,3-22,3)$ & $41,4(38,1-44,7)$ & \\
\hline Ocasional & $80,3(77,7-82,7)$ & $58,6(55,3-61,9)$ & \\
\hline Sexo & & & 0,01 \\
\hline Masculino & $40,1(37,5-42,6)$ & $44,8(42,9-46,7)$ & \\
\hline Feminino & $59,9(57,4-62,5)$ & $55,2(53,3-57,2)$ & \\
\hline Autopercepção de saúde bucal & & & $<0,001$ \\
\hline Ótima & $10,7(8,9-12,9)$ & $16,2(14,5-18,1)$ & \\
\hline Boa & $35,3(32,5-28,2)$ & $49,6(47,1-52,1)$ & \\
\hline Regular & $38,9(36,1-41,9)$ & $27,7(25,3-30,2)$ & \\
\hline Ruim/Péssima & $15,1(12,9-17,6)$ & $6,5(5,4-7,8)$ & \\
\hline Recebeu orientação sobre prevenção & & & $<0,001$ \\
\hline Não & $40,2(37,1-43,3)$ & $21,1(18,6-23,7)$ & \\
\hline Sim & $59,8(56,7-62,9)$ & $78,9(76,3-81,4)$ & \\
\hline \multicolumn{4}{|l|}{ Dentista recomenda mais tratamento do } \\
\hline que eu preciso & & & $<0,001$ \\
\hline Concordo & $29,6(26,5-32,9)$ & $21,7(19,4-24,2)$ & \\
\hline Não concordo & $50,8(47,1-54,5)$ & $66,0(62,8-69,0)$ & \\
\hline Não sei & $19,6(16,8-22,8)$ & $12,3(10,5-14,3)$ & \\
\hline \multicolumn{4}{|c|}{ Todo mundo perde os dentes com o passar } \\
\hline do tempo & & & $<0,001$ \\
\hline Concordo & $53,3(50,0-56,7)$ & $38,2(35,4-41,3)$ & \\
\hline Não concordo & $39,3(36,3-42,4)$ & $54,6(51,5-57,6)$ & \\
\hline Não sei & $7,4(5,7-9,5)$ & $7,2(6,0-8,6)$ & \\
\hline \multicolumn{4}{|c|}{ Salvar os dentes da frente é mais importante } \\
\hline que salvar os dentes de trás & & & $<0,001$ \\
\hline Concordo & $59,8(56,5-63,1)$ & $48,2(45,2-51,3)$ & \\
\hline Não concordo & $35,3(31,9-38,7)$ & $47,0(44,0-50,0)$ & \\
\hline Não sei & $4,9(3,7-6,5)$ & $4,8(3,9-6,0)$ & \\
\hline Necessidade de tratamento & & & $<0,001$ \\
\hline Sim, para revisão & $21,3(18,5-24,5)$ & $23,1(20,8-25,5)$ & \\
\hline Não, está ok & $34,3(31,5-37,2)$ & $43,2(40,7-45,9)$ & \\
\hline Sim, tenho problemas & $34,6(31,6-37,8)$ & $24,1(21,9-26,4)$ & \\
\hline Não, pode esperar & $9,8(8,0-12,0)$ & $9,6(8,2-11,1)$ & \\
\hline \multicolumn{4}{|l|}{ 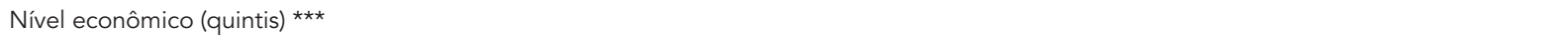 } \\
\hline Q1 (mais pobres) & $37,6(33,1-42,3)$ & $10,8(8,7-13,4)$ & $<0,001 \#$ \\
\hline Q2 & $28,7(24,9-32,8)$ & $19,1(16,4-22,2)$ & \\
\hline Q3 & $19,4(16,1-23,2)$ & $18,6(16,1-18,9)$ & \\
\hline Q4 & $10,4(7,9-13,4)$ & $23,8(21,0-32,9)$ & \\
\hline Q5 (mais ricos) & $4,0(2,6-6,1)$ & $27,6(22,9-32,9)$ & \\
\hline
\end{tabular}

IC95\%: intervalo de $95 \%$ de confiança.

* Categoria inclui serviços conveniados e particulares;

** Teste de heterogeneidade;

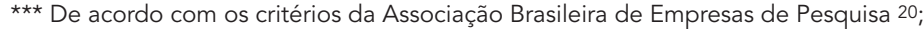

\# Teste de tendência linear. 


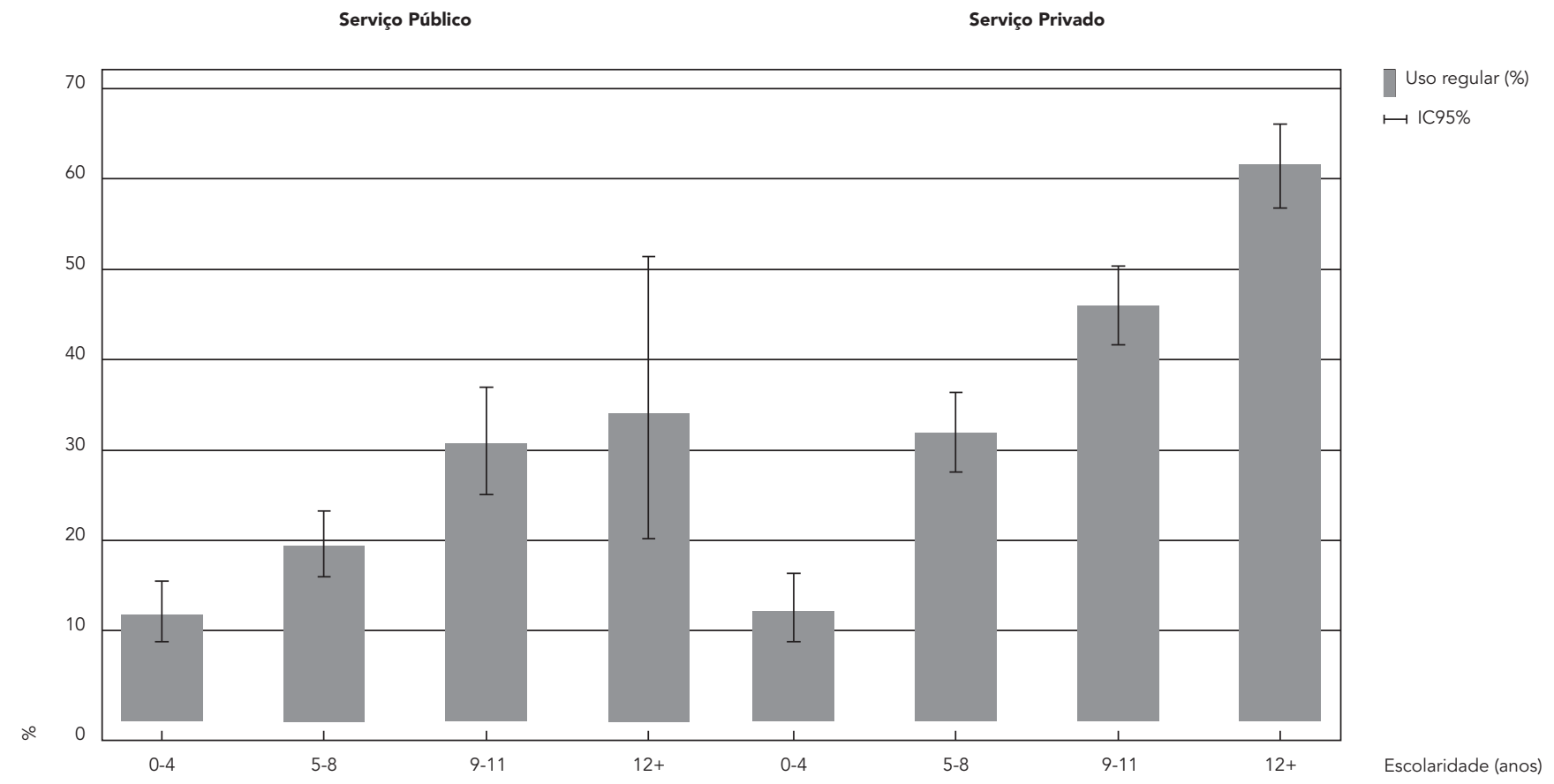

Teste de tendência linear: serviço público $(p<0,001)$; serviço privado $(p<0,001)$.

sem companheiro, usuários de serviços privados, maior escolaridade e maior nível econômico. Opinião favorável em relação ao profissional e conhecimentos sobre prevenção também foram associados a maior utilização de forma regular. Indivíduos que responderam sim, com o objetivo de fazer uma revisão, e não, com a justificativa de que estava tudo bem, tiveram maior prevalência de uso regular quando comparados àqueles que disseram não, pois apesar de terem um problema para resolver, este poderia esperar.

Não permaneceu estatisticamente significativa, após ajuste para confundimento, a associação com a cor da pele $(p=0,32)$; o mesmo se deu com não ser fatalista em relação ao edentulismo ( $p=0,72)$ e não dar maior importância ao componente estético da saúde bucal $(p=0,56)$.

\section{Discussão}

Este é um estudo de base populacional, representativo da população adulta da cidade de Pelotas, no qual se conseguiu manter o percentual de perdas e recusas em níveis muito reduzidos. Ao se estudar a utilização regular de serviços odontológicos, devem-se incluir indivíduos que usaram e que não usaram os serviços, o que se enquadra adequadamente em estudos de base populacional. Contudo, isso leva a uma possível limitação, em virtude do fato de que o desfecho tem que ser medido com base no auto-relato de consultas odontológicas. Levando-se em consideração que o uso de serviços odontológicos de forma regular é um comportamento desejável socialmente, alguns indivíduos podem superestimar suas consultas com o cirurgião-dentista, ou privilegiar o uso preventivo como justificativa. É pouco provável que ocorra o contrário, ou seja, que indivíduos subestimem as suas consultas, ou apresentem como razão da consulta um problema, quando na verdade esta foi o uso regular.

A definição de uso regular de serviços odontológicos neste estudo não levou em conta a 
Tabela 3

Análise multivariável conforme modelo conceitual do uso regular de serviços odontológicos em adultos, segundo as variáveis de predisposição, facilitação e necessidade ( $\mathrm{N}=2.961)$. Pelotas, Rio Grande do Sul, Brasil, 2008.

\begin{tabular}{|c|c|c|c|}
\hline Nível & Variáveis & RP (IC95\%) & Valor de $p$ \\
\hline \multirow[t]{21}{*}{1 * } & Sexo * & & $<0,001$ \\
\hline & Masculino & 1,00 & \\
\hline & Feminino & $1,22(1,11-1,33)$ & \\
\hline & Idade (anos) ** & & $<0,001 \# \# \#$ \\
\hline & 20-39 & $1,60(1,32-1,95)$ & \\
\hline & $40-59$ & $1,42(1,16-1,74)$ & \\
\hline & 60 anos & 1,00 & \\
\hline & Escolaridade (anos) $* *$ & & $<0,001 \# \# \#$ \\
\hline & $0-4$ & 1,00 & \\
\hline & $5-8$ & $1,93(1,50-2,47)$ & \\
\hline & $9-11$ & $2,58(2,03-3,28)$ & \\
\hline & 12 & $3,39(2,61-4,40)$ & \\
\hline & Situação conjugal ** & & 0,01 \\
\hline & Com companheiro & 1,00 & \\
\hline & Sem companheiro & $1,14(1,03-1,27)$ & \\
\hline & Nível econômico (quintis) ${ }^{\star \star \star}, \#$ & & $<0,001 \# \# \#$ \\
\hline & Q1 (mais pobres) & 1,00 & \\
\hline & $\mathrm{Q} 2$ & $1,44(1,14-1,70)$ & \\
\hline & Q3 & $1,63(1,28-2,10)$ & \\
\hline & Q4 & $1,86(1,45-2,39)$ & \\
\hline & Q5 (mais ricos) & $1,95(1,51-2,50)$ & \\
\hline \multirow[t]{9}{*}{2 * } & Tipo de serviço que procura \# & & $<0,001$ \\
\hline & Público & 1,00 & \\
\hline & Particular & $1,34(1,14-1,56)$ & \\
\hline & Convênio & $1,45(1,23-1,72)$ & \\
\hline & Autopercepção de saúde bucal \#\# & & $<0,001 \# \# \#$ \\
\hline & Otima & $3,05(2,00-4,66)$ & \\
\hline & Boa & $2,25(1,47-3,43)$ & \\
\hline & Regular & $1,59(1,02-2,46)$ & \\
\hline & Ruim/Péssima & 1,00 & \\
\hline \multirow[t]{12}{*}{3 * } & Recebeu orientação sobre prevenção ** & & $<0,001$ \\
\hline & Não & 1,00 & \\
\hline & $\operatorname{Sim}$ & $2,08(1,67-2,59)$ & \\
\hline & Dentista recomenda mais tratamento do que eu preciso ** & & $<0,001$ \\
\hline & Concordo & 1,00 & \\
\hline & Não concordo & $1,19(1,04-1,37)$ & \\
\hline & Não sei & $0,74(0,59-0,93)$ & \\
\hline & Autopercepção de necessidade de tratamento \#\# & & $<0,001$ \\
\hline & Sim, para uma revisão & $1,58(1,24-2,01)$ & \\
\hline & Não, está tudo bem com a minha boca & $1,29(1,02-1,64)$ & \\
\hline & Sim, tenho dor ou problemas para resolver & $1,15(0,90-1,48)$ & \\
\hline & Não, embora eu tenha um problema ele pode esperar & 1,00 & \\
\hline
\end{tabular}

RP: razão de prevalência; IC95\%: intervalo de 95\% de confiança.

* Níveis do modelo hierárquico de análise utilizado. As variáveis estão ajustadas para as do mesmo nível e dos níveis acima;

** Variáveis de predisposição segundo classificação de Andersen \& Newman;

*** De acordo com os critérios da Associação Brasileira de Empresas de Pesquisa 20;

\# Variáveis de facilitação segundo classificação de Andersen 18 e Andersen \& Newman 19;

\#\# Variáveis de necessidade segundo classificação de Andersen 18 e Andersen \& Newman 19;

\#\#\# Teste de tendência linear. 
freqüência de consultas, diferenciando os indivíduos que só procuram os serviços quando estão com dor ou precisam resolver uma necessidade imediata daqueles que procuram assistência quando ainda assintomáticos, com intuito de fazer uma revisão 16,22. Embora uma alta freqüência de visitas ao dentista possa resultar em sobretratamento ou iatrogenia, esse não é um problema da população brasileira como um todo, que sofre ainda com a falta de acesso aos serviços odontológicos 2 .

O instrumento foi elaborado pelos autores; quanto à questão utilizada para definição do desfecho, esta foi anteriormente aplicada por Gilbert et al. 16. Essa questão foi traduzida e pré-testada entre indivíduos usuários de serviços públicos de saúde para verificar o seu entendimento. Posteriormente, foi testada em um estudo-piloto com 117 indivíduos. A questão foi aplicada antes de qualquer outra sobre utilização de serviço odontológico, a fim de se evitar que o indivíduo captasse o interesse específico na utilização regular, o que poderia induzir sua resposta.

Algumas associações encontradas devem ser interpretadas com cautela, pois, sendo este um estudo transversal, pode ocorrer viés de causalidade reversa. Usando esse tipo de delineamento, não é possível saber se os indivíduos usam os serviços de forma regular porque tiveram orientação sobre prevenção e acreditam ser esta prática benéfica para manutenção da saúde bucal ou se, por usarem os serviços regularmente, obtiveram maior conhecimento sobre como prevenir doenças bucais. Outras variáveis, como autopercepção de saúde, confiança no profissional, crenças sobre tratamento e autopercepção de necessidade de tratamento, também são passíveis do mesmo tipo de viés. Entretanto, neste estudo, o principal objetivo não foi identificar fatores causais, e sim variáveis que seriam marcadores individuais do uso dos serviços de forma regular, as quais pudessem ser trabalhadas a fim de incrementar esse tipo de utilização.

A prevalência, neste estudo, de uso regular de serviços odontológicos foi semelhante à encontrada pelo inquérito nacional de saúde bucal em 2004 (Projeto SB Brasil 2003) 23, no qual 31,9\% dos indivíduos do Estado do Rio Grande do Sul, que consultaram no último ano, relataram ser a revisão o motivo da última consulta ao cirurgiãodentista. Em Bambuí, Minas Gerais, um inquérito populacional 14 encontrou prevalência de uso regular de $24,6 \%$. No estudo mineiro foi considerado usuário regular o indivíduo que fez pelo menos uma consulta no último ano, sendo a revisão o principal motivo. Pesquisas em outros países encontraram prevalências muito variáveis, de $18 \%$ a $64 \% 16,24,25$. Essa variabilidade se deve a grandes diferenças na organização dos serviços, na população em estudo e nos distintos critérios de definição de usuários regulares.

Considerando a metodologia utilizada e o percentual de perdas e recusas, este estudo é representativo da população com 20 anos ou mais de idade da zona urbana de Pelotas. Extrapolando os dados para a população, aproximadamente 150 mil indivíduos de 220 mil com 20 anos de idade procurariam os serviços odontológicos somente quando tivessem dor ou algum desconforto, fato que poderia interferir na qualidade de vida das pessoas, sendo motivo de impedimentos de atividades cotidianas 26 .

Em relação às variáveis de predisposição, como sexo e idade, foram encontrados resultados semelhantes aos de diversos autores. As mulheres 8,10,20,27, assim como indivíduos mais jovens, com idade entre 20-39 e 40-59 anos, comparados aos com 60 anos ou mais ${ }^{3}$, fizeram maior uso regular de serviços odontológicos. Ter não só serviços preparados para suprir as necessidades dessa população 27, como também facilitação do acesso, poderia contribuir para maior captação de homens e idosos. A utilização dos serviços médicos por adultos e idosos é muito superior ao uso que estes fazem de serviços odontológi$\cos 2,28$, portanto o profissional médico poderia ser um valioso aliado no incentivo ao uso dos serviços odontológicos, o que, além de monitorar problemas dentários e próteses, teria um importante papel na detecção precoce do câncer de boca 25,29 .

A maior utilização por mulheres desaparece entre jovens de 20-39 anos de idade. Uma possível explicação para isso seria um efeito de coorte, em que os homens estariam utilizando mais os serviços de forma preventiva, seja por maior valorização à saúde, seja por estética. Por outro lado, poderia ser um efeito exclusivo da idade, isto é, os jovens utilizariam mais os serviços, mas, com passar dos anos, esta utilização tenderia a diminuir. Para melhor compreender esse fato, estudos longitudinais seriam necessários.

Conhecimentos sobre as doenças, crenças sobre tratamentos, atitudes em relação ao profissional e valor dado à saúde são importantes determinantes de doenças bucais, de uso dos serviços odontológicos e de autocuidado ${ }^{30}$. Mesmo após controle para as variáveis sócio-econômicas e demográficas, os indivíduos que tinham maior conhecimento sobre a doença e demonstravam atitudes positivas tiveram maior chance de fazer uso dos serviços de forma regular. Ao se controlar para variáveis do nível anterior e para as do mesmo nível, algumas perderam a significância, talvez porque todas essas variáveis sobre conhecimentos traduzam um mesmo domínio, per- 
manecendo no modelo aquela que tinha maior magnitude de efeito na análise bruta.

Com a metodologia quantitativa utilizada e com o número limitado de questões de que se dispunha, tornou-se complexa a quantificação de questões subjetivas relacionadas a crenças e opiniões sobre o profissional, assim como de experiências relacionadas com tratamentos e doenças bucais. Não obstante, mesmo de forma limitada, tentamos entender como essas variáveis poderiam se relacionar com a utilização dos serviços de forma regular, uma vez que condição econômica favorável e acesso facilitado aos serviços, per se, não garantem este tipo de utilização.

Dentre as variáveis de facilitação, neste estudo, foram avaliados nível econômico e tipo de serviço procurado, ambos mostrando-se associados com o desfecho. No modelo de análise, incluíram-se o nível econômico (facilitação) e a escolaridade (predisposição), por se entender que ambos têm significados diferentes, ao contrário do que se observa no estudo realizado por Matos et al. 14, em Bambuí, no qual se optou por incluir apenas uma das variáveis. Enquanto nível econômico traduz a capacidade de compra dos serviços, escolaridade traduz a consciência, o entendimento de que, para prevenir doenças bucais, as consultas de rotina são importantes. Os resultados confirmam a hipótese, uma vez que, após ajuste, ambas continuaram altamente significativas. No entanto, o efeito bruto de cor da pele desapareceu após o controle para essas variáveis.

A variável de necessidade avaliada neste estudo foi a autopercepção de necessidade de tratamento. Semelhante ao observado por outros autores 20,30 , a maior prevalência de uso de forma regular se deu entre aqueles que relataram não necessitar de tratamento por estar tudo bem e aqueles que disseram sim, por necessidade de se fazer uma consulta de rotina, quando comparados com aqueles conscientes de que tinham um problema, mas cuja resolução poderia ser adiada.

Importante função do SUS é diminuir as desigualdades (princípio da eqüidade), papel que, na situação em estudo, parece não estar sendo cumprido. Primeiramente porque, entre todos os serviços, o SUS teve a menor prevalência de utilização de forma regular. Idealmente se esperaria uma prevalência de uso mais equânime entre os serviços. Em segundo lugar, quando analisamos as prevalências de uso regular entre aqueles que procuram o serviço público e o serviço privado (particular +convênio), podemos verificar que a diferença de utilização entre os mais e os menos escolarizados é menor no grupo dos usuários do serviço público, quando comparados aos do serviço privado. Essa diferença, entretanto, deu-se por existir menor prevalência entre aqueles mais escolarizados, e não por ter havido maior uso entre aqueles menos favorecidos. A nosso ver, o serviço público não pode ter uma atitude de espera pela demanda, sendo necessária a busca ativa daqueles que mais necessitam de atenção, criando estratégias para captá-los, a fim de priorizar a população mais necessitada 31 .

Algumas das características de predisposição apresentam potencial para serem modificadas 17 , podendo, portanto, ser trabalhadas pelos profissionais da área como as que envolvem conhecimentos, crenças e atitudes em relação à doença, ao tratamento, aos profissionais e ao valor que se dá à saúde. Instrumentalizar os indivíduos pode ser a chave para a mudança de comportamentos. Abordagens que estimulassem os usuários de serviços públicos e privados a valorizar ações preventivas, a estabelecer vínculo com o serviço e seus profissionais, a obter maior conhecimento sobre o processo saúde-doença e a usar os serviços de forma regular deveriam ser enfatizadas nas ações dos serviços de saúde bucal.

Nosso estudo mostrou que há menor uso regular de serviço odontológico entre os usuários de serviços públicos, e que, entre estes, o uso regular é menor entre os mais pobres e menos escolarizados. Isso sugere que deve investir-se, na esfera das políticas públicas, no estímulo ao uso regular. Os caminhos, como já citamos anteriormente, são principalmente aqueles ligados à informação do paciente, à construção de uma relação de confiança e à adoção de uma atitude proativa no contato com o paciente, não esperando que este tome a iniciativa da procura do serviço, numa situação de necessidade imediata. 


\section{Resumo}

O objetivo deste estudo foi avaliar o uso regular de serviços odontológicos entre adultos, identificando grupos nos quais esse comportamento é mais freqüente. O estudo foi realizado em Pelotas, Rio Grande do Sul, Brasil, com desenho transversal de base populacional, envolvendo 2.961 indivíduos, que responderam um questionário estruturado. A prevalência de uso regular encontrada foi de 32,8\%. Estiveram positivamente associadas ao uso de forma regular as seguintes características: ser do sexo feminino, ter idade $\leq 60$ anos, não ter companheiro, ter alta escolaridade, ter maior nível econômico, usar serviços privados, ter autopercepção de saúde bucal boalótima, não ter autopercepção de necessidade de tratamento, ter recebido orientação sobre prevenção e ter manifestado opinião favorável ao profissional. O uso regular foi pouco freqüente, especialmente entre os menos favorecidos (15\%). Restringindo a análise apenas para usuários do serviço público, observou-se maior utilização regular entre os mais escolarizados. Os serviços de saúde, em especial o público, devem trabalhar a fim de incrementar o uso regular e preventivo.

Assistência Odontológica; Serviços de Saúde; Adulto

\section{Colaboradores}

M. B. J. Camargo foi responsável pela concepção do estudo, realizou a revisão de literatura, realizou a maior parte da análise dos dados e redigiu o texto. S. C. Dumith participou da elaboração do artigo e colaborou nas análises. A. J. D. Barros contribuiu com a concepção do estudo e supervisionou todas as suas etapas, participou da redação do artigo e análise dos dados.

\section{Agradecimentos}

À Coordenação de Aperfeiçoamento de Pessoal de Nível Superior (CAPES).

\section{Referências}

1. Narvai PC, Frazão P, Roncalli AG, Antunes JLF, Camargos P, Ribeiro Y, et al. Cárie dentária no Brasil: declínio, polarização, iniqüidade e exclusão social. Rev Panam Salud Pública 2006; 19:385-93.

2. Barros AJD, Bertoldi AD. Desigualdades na utilização e no acesso a serviços odontológicos: uma avaliação em nível nacional. Ciênc Saúde Coletiva 2002; 7:709-17.

3. Araújo YP, Dimenstein M. Estrutura e organização do trabalho do cirurgião-dentista no PSF de municípios do Rio Grande do Norte. Ciênc Saúde Coletiva 2006; 11:219-27.

4. Chaves SCL, Vieira-da-Silva LM. Atenção a saúde bucal e a descentralização da saúde no Brasil: estudo de dois casos exemplares no Estado da Bahia. Cad Saúde Pública 2007; 23:1119-31.

5. Santos AM, Assis MMA. Da fragmentação à integralidade: construindo e (des)construindo a prática de saúde bucal no Programa de Saúde da Família (PSF) de Alagoinhas, BA. Ciênc Saúde Coletiva 2006; 11:53-61.
6. Gilbert GH, Stoller EP, Duncan RP, Earls JL, Campbell AM. Dental self-care among dentate adults: contrasting problem-oriented dental attenders and regular dental attenders. Spec Care Dentist 2000; 20:155-63.

7. Beirne P, Forgie A, Clarkson JE, Worthington HV. Recall intervals for oral health in primary care patients. (Cochrane Review). In: The Cochrane Library, Issue 4, 2007. Oxford: Update Software.

8. Afonso-Souza G, Nadanovsky P, Chor D, Faerstein E, Werneck GL, Lopes CS. Association between routine visits for dental checkup and self-perceived oral health in an adult population in Rio de Janeiro: the Pró-Saúde Study. Community Dent Oral Epidemiol 2007; 35:393-400.

9. Alexandre GC, Nadanovsky P, Lopes CS, Faerstein E. Prevalência e fatores associados à ocorrência da dor de dente que impediu a realização de tarefas habituais em uma população de funcionários públicos no Rio de Janeiro, Brasil. Cad Saúde Pública 2006; 22:1073-8. 
10. Lawrence HP, Thomson WM, Broadbent JM, Poulton R. Oral health-related quality of life in a birth cohort of 32-year olds. Community Dent Oral Epidemiol 2008; 36:305-16.

11. Mullally BH, Linden GL. The periodontal status of irregular dental attenders. J Clin Periodontol 1994; 21:544-8.

12. Celeste RK, Nadanovsky P, Ponce de Leon A. Associação entre procedimentos preventivos no serviço público de odontologia e a prevalência de cárie dentária. Rev Saúde Pública 2007; 41:830-8.

13. Gilbert GH, Shelton BJ, Duncan RP. Use of specific dental treatment procedures by dentate adults during a 24-month period. Community Dent Oral Epidemiol 2002; 30:260-76.

14. Matos DL, Lima-Costa MFF, Guerra HL, Marcenes W. Projeto Bambuí: estudo de base populacional dos fatores associados com o uso regular de serviços odontológicos em adultos. Cad Saúde Pública 2001; 17:661-8.

15. Barros AJD, Menezes AMB, Santos IS, Assunção MCF, Gigante D, Fassa AG, et al. O Mestrado do Programa de Pós-graduação em Epidemiologia da UFPel baseado em consórcio de pesquisa: uma experiência inovadora. Rev Bras Epidemiol 2008; 11 Suppl 1:133-44.

16. Gilbert GH, Duncan RP, Heft MW, Coward RT. Dental health attitudes among dentate black and white adults. Med Care 1997; 35:255-71.

17. Andersen RM. Revisiting the behavioral model and access to medical care: does it matter? J Health Soc Behav 1995; 36:1-10.

18. Andersen RM, Newman JF. Societal and individual determinants of medical care utilization in the United States. Milbank Mem Fund Q Health Soc 1973; 51:95-124.

19. Associação Brasileira de Empresas de Pesquisa. Critério de classificação econômica Brasil. http:// www.abep.org/codigosguias/CCEB2008-Base 2006e2007.pdf (acessado em 10/Abr/09).

20. Heft MW, Gilbert GH, Shelton BJ, Duncan RP. Relationship of dental status, sociodemographic status, and oral symptoms to perceived need for dental care. Community Dent Oral Epidemiol 2003; 31:351-60.
21. Barros AJD, Hirakata VN. Alternatives for logistic regression in cross-sectional studies: an empirical comparison of models that directly estimate the prevalence ratio. BMC Med Res Methodol 2003; $3: 21$.

22. Gilbert GH, Duncan RP, Vogel WB. Determinants of dental care use in dentate adults: six-monthly use during a 24-month period in the Florida Dental Care Study. Soc Sci Med 1998; 47:727-37.

23. Coordenação Nacional de Saúde Bucal, Departamento de Atenção Básica, Secretaria de Atenção à Saúde, Ministério da Saúde. Projeto SB Brasil 2003. Condições de saúde bucal da população brasileira 2002-2003. Brasília: Ministério da Saúde; 2004.

24. Taani DQ. Periodontal awareness and knowledge, and pattern of dental attendance among adults in Jordan. Int Dent J 2002; 52:94-8.

25. Tickle M, Worthington HV. Factors influencing perceived treatment need and the dental attendance patterns of older adults. Br Dent J 1997; 182: 96-100.

26. Lacerda JT, Simionato EM, Peres KG, Peres MA, Traebert J, Marcenes W. Dor de origem dental como motivo de consulta odontológica em uma população adulta. Rev Saúde Pública 2004; 38:453-8.

27. Matos D, Giatti L, Lima-Costa M. Fatores sócio-demográficos associados ao uso de serviços odontológicos entre idosos brasileiros: um estudo baseado na Pesquisa Nacional por Amostra de Domicílios. Cad Saúde Pública 2004; 20:1290-7.

28. Pinheiro RS, Viacava F, Travassos C, Brito AS. Gênero, morbidade, acesso e utilização de serviços de saúde no Brasil. Ciênc Saúde Coletiva 2002; 7:687-707.

29. Benedetti TRB, Mello A, Goncalves LHT. Idosos de Florianópolis: autopercepção das condições de saúde bucal e utilização de serviços odontológicos. Ciênc Saúde Coletiva 2007; 12:1683-90.

30. Riley 3rd JL, Gilbert GH, Heft MW. Dental attitudes: proximal basis for oral health disparities in adults. Community Dent Oral Epidemiol 2006; 34:289-98.

31. Souza TMS, Roncalli AG. Saúde bucal no Programa Saúde da Família: uma avaliação do modelo assistencial. Cad Saúde Pública 2007; 23:2727-39.

Recebido em 17/Nov/2008

Versão final reapresentada em 16/Abr/2009

Aprovado em 09/Jun/2009 